\title{
Radar target hardware-in-the-loop simulation in carrier frequencies for SAR tests
}

\author{
Tatiana A. Lepekhina and Vadim I. Nikolaev* \\ Joint Stock Company "Radio Engineering Corporation "Vega”, 121170 Moscow, Russia
}

\begin{abstract}
Methods of hardware-software simulation of test scenes of varying complexity are proposed for synthetic aperture space radar (SAR) ground tests and testing its components, as well as for checking its end-toend characteristics and calibration during flight tests. The principles of designing and features of the hardware implementation of an active simulator of radar targets are described. Some methods for solving problems associated with the synchronization of received and generated signals are proposed.
\end{abstract}

The world development of remote Earth sensing technologies by means of synthetic aperture radars (SAR) is related to the increased variety of tasks solved with their help, and the list of requirements to the characteristics and functions of equipment, software and algorithmic support and methods of their control [1,2]. Taking into consideration the variety of survey modes and the increased share of information products obtained using onboard computing means, while creating a system of space SAR ground tryout it is necessary to provide the means of flightless ground tests to ensure hardware-in-the-loop simulation of objects, motion of the satellite carrier and automated analysis of the experiment results to evaluate the performance of SAR. The necessary condition for the automation of the experiment is the use of test actions with calibrated characteristics relevant to the SAR tested parameters, the possibility of multiple repetition of the experiment with preserving constant conditions and using criteria suitable for the automation of analysis techniques. A system approach to the development of space SAR demands creating uniform techniques for ground and flight tests. In ground tests, synchronization between the SAR and the simulator can be achieved by using cable or wireless communication lines. During flight tests and at the stage of regular operation, the task of synchronizing them by the sounding signal becomes urgent.

The simplest type of a simulated radar target is a single point target. Simulation of point targets is carried out with the help of transponders having a fiber-optic or digital delay line. Currently, such devices are used for radiometric calibration of space SAR, and there are also known examples of including a delay line in the radar as a means of integrated efficiency control. This is the way to simulate the signal reflected from a single point target on the traverse; here for the image synthesis one should use a simplified algorithm, which excludes changes in the distance between the phase center of the antenna and the target when the carrier is moving. Despite the simplified law of conversion, this method of

*Corresponding author: vnikolaev6503@gmail.com 
simulating a reflected signal allows obtaining the hardware response function of the SAR for operational control of its efficiency and preliminary assessment of the resolution on the shape and width of the main lobe, and the side lobes levels. Simulation of a group of point targets forming a figure on a plane (usually a square) allows identifying factors affecting the type of the response function for point targets located not on the traverse: this may be nonorthogonality of the demodulator quadrature, as well as nonlinearity of the signal transmission in the video path, for example, due to amplitude overload.

Another, no less important, task is to simulate complex targets, taking into account the law of changing the distance to point targets, which is close to real in flight conditions, in order to be able to use the standard algorithm for compressing the radio hologram. Methods of simulation, as well as subsequent synthesis and analysis of radar images of simulated targets for flight tests are similar to ground ones, except for some features. When simulating ground tests, the signal is converted taking into account the model of mutual motion of the SAR antenna and all the elements of the simulated radar test pattern, while with the SAR working in orbit, the conversion is based on the difference component of the laws of motion for the elements of the simulated radar test pattern shifted relative to the location of the transponder.

To describe the principle of hardware-in-the-loop (HIL) simulation of test impacts, it is necessary to give a brief mathematical description of the process of arbitrary scene survey by means of SAR.

The scene to be surveyed is described as a two-dimensional field of complex backscattering coefficient in the tested SAR frequency range:

$$
\dot{s}(x, r)=\sqrt{\sigma_{0}(x, r)} \cdot e^{i \varphi(x, r)},
$$

where $\sigma_{0}$ is the specific radar cross-section (SRCS) of the surveyed scene at the point with coordinates $(\mathrm{x}, \mathrm{r}), \varphi$ is the reflected signal initial phase shift, or as a set of $\mathrm{N}$ discrete reflectors with different backscattering coefficients,

$$
\dot{s}(x, y, z)=\sum_{m=1}^{N} \dot{s}_{m} \delta\left(x_{m}, y_{m}, z_{m}\right),
$$

where $\left(x_{m}, y_{m}, z_{m}\right)$ are the coordinates of the m-th discrete reflector, $\dot{s}_{m}=\sqrt{\sigma_{m}} e^{i \varphi_{m}}$ is its complex backscattering coefficient, $\sigma_{m}$ and $\varphi_{m}$ are its RCS and reflected signal phase shift.

Based on the foregoing, a technique is proposed for the simulation of a reflected signal.

The formation of a reflected signal from the surveyed area is the result of three processes:

1) the generation of a sounding signal (SS) being a sequence of modulated pulses $\dot{u}_{\text {snd }}(t, \tau)$, where $\tau$ is the "fast" time (the argument of complex functions that describe the laws of intrapulse modulation of the sounding and reflected signals), $t$ is the "slow" time, which describes the motion of the carrier in orbit;

2) the reflection of the SS from each element of the surveyed scene with the complex backscattering coefficient $\dot{s}(x, r)$;

3 ) the formation of the reflected signal as a superposition of elementary reflected signals, each having the total delay in the propagation on the down and up lines:

$$
\dot{u}_{t r}(t, \tau)=\sum_{m=1}^{N} L_{m}^{2}(t) \cdot e^{-j \frac{4 \pi}{\lambda} \rho\left(t, x_{m}, y_{m}, z_{m}\right)} \cdot \dot{s}_{m} \dot{u}_{s n d}\left(\tau-\frac{2 \rho\left(t, x_{m}, y_{m}, z_{m}\right)}{c}\right),
$$


where $\rho$ is the distance between the satellite and the m-th elementary reflector at the moment of "slow" time t.

Such superposition can be represented as a convolution of the complex envelope of the sounding pulse with some impulse response

$$
\dot{u}_{t r}(t, \tau)=\dot{u}_{s n d}\left(t, \tau^{\prime}\right) * \dot{h}_{t r}\left(t, \tau-\tau^{\prime}\right),
$$

where for simulation in ground tests

$$
\dot{h}_{t r}\left(t, \tau^{\prime}\right)=\sum_{m=1}^{N} L_{m}^{2}(t) \cdot e^{-j \frac{4 \pi}{\lambda} \rho\left(t, x_{m}, y_{m}, z_{m}\right)} \cdot \dot{s}_{m} \delta\left(\tau^{\prime}-\frac{2 \rho\left(t, x_{m}, y_{m}, z_{m}\right)}{c}\right),
$$

and to simulate complex targets when interacting with a satellite in orbit, the variable distance in formula (4) is replaced by $\rho^{\prime}\left(t, x_{m}, y_{m}, z_{m}\right)=\rho\left(t, x_{m}, y_{m}, z_{m}\right)-\rho\left(t, x_{0}, y_{0}, z_{0}\right)$, where $\left(x_{0}, y_{0}, z_{0}\right)$ are the simulator coordinates.

Active simulation of radar test patterns during flight tests and regular operation of space SAR is widely used for radiometric calibration, measurement of polarimetric characteristics $[3,4]$, and is also considered as a feasible method for generating test effects for experimental determination of radiometric resolution and dynamic range from distributed targets [5].

To assess SAR radiometric resolution in distributed target survey, as well as to test the functions of automated image decryption (object recognition), it is necessary to simulate more complex multipoint targets and arbitrary scenes with a number of elements of $10^{7}$ or more [5]. For medium resolution modes and short length of the simulated scene in terms of range (the total number of samples of the digitized pulse of the sounding signal and pixels of the complex target model does not exceed 2048), convolutional signal conversion in real time is implemented according to the algorithm described in [6]. More complex models use tryout techniques with advanced data preparation, requiring preliminary recording of the SAR sounding signal and executing a subsequent control session in the same mode, with a simulated reflected signal fed to the input of the SAR receiver modeled according to the data of the previously recorded SS. Both modes require synchronization of the cyclograms of the SAR and the simulator using trigger signals and synchronization of the reference frequencies of both devices.

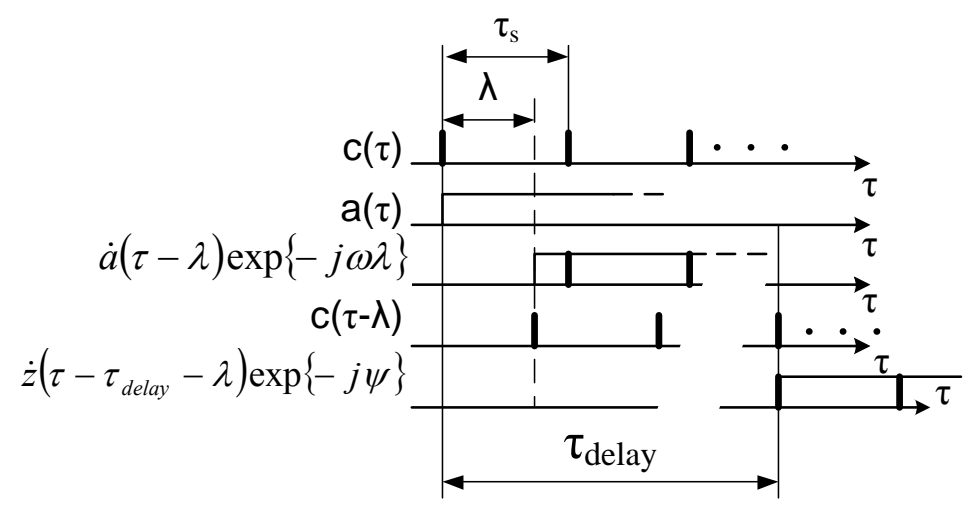

Fig. 1. The cycle diagram of the formation of the return signal with synchronization from the sounding pulse.

The cyclogram of the formation of the return signal with synchronization from the sounding pulse is shown in Fig. 1. The start of the cycle is the calculated moment of the 
conditional start of the sounding pulse ti, not necessarily coinciding with the front of the pulse amplitude envelope, but tied to the front of the clock pulse of the receiver channel ADC [7]. The programmed delay $\tau_{\text {delay }}$ of the start of the transmission pulse generation with respect to the moment $\left(t_{i}+\lambda\right)$ of the conditional start of the received sounding pulse is set equal to an integer number of clock cycles and must be maintained throughout the session. To provide this, a sequence of clock pulses of the transmitter channel DAC is formed, delayed relative to the ADC clock by $\lambda$ (at $\lambda>0$ ) or by $\tau_{\mathrm{s}}-\lambda$ (at $\lambda<0$ ).

A correlation analyzer with minimal delay after receiving a sounding pulse computes in parallel the convolution of its complex envelope with two preloaded reference functions. The first of them is the time-inverted complex conjugate envelope of the expected pulse, and the convolution result is an approximation of its autocorrelation function (ACF). The result of the second convolution is a discriminatory characteristic (DC), which takes a zero value with an argument corresponding to the maximum of the first convolution (Fig. 2). In this case, it should be taken into account that, as a rule for modulating pulses of sounding signals in radar, there are selected functions, whose autocorrelation function has one dominant main lobe and low levels of side lobes. Using this property, correlation-extremal algorithms known from the theory of automatic control and navigation [8] can be applied to determine the time offset of the received sounding pulse.

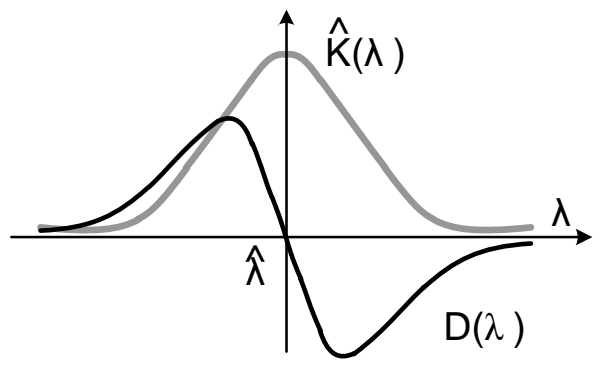

Fig. 2. Determination of the delay and initial phase of the received impulse based on the discriminatory characteristic.

The processing of the received sounding impulse is as follows.

The received sounding signal can be represented by

$$
\dot{s}_{i}(\tau)=\dot{a}_{i}\left(\tau-\tau_{i}\right) \cdot \exp \left\{-j \omega \tau_{i}\right\}
$$

where $\tau_{i}=\tau_{0 i}+\lambda_{i}, \dot{a}_{i}(t)$ is the sounding impulse complex envelope, $\tau_{i}$ is the time from the received signal recording start moment taken as the sounding cycle start, $\omega$ is the signal carrier frequency, $\tau_{0 i}$ is the estimated delay of the beginning of the received pulse from the beginning of the cycle, $\lambda_{i}$ is the random component of the delay in receiving the $\mathrm{i}$-th pulse in the session. After demodulation and analog-to-digital conversion, this signal is written to the buffer RAM of the signal processor in a discrete complex form:

$$
\dot{\xi}_{i k}=\dot{\xi}_{i}\left(k \tau_{s}\right)=\dot{s}_{i}\left(k \tau_{s}-\tau_{0}-\lambda_{i}\right) \cdot \exp \left\{-j \psi_{i}\right\}+\dot{n}_{i}\left(k \tau_{s}\right)
$$

where $\tau_{s}$ is the sampling period, $\lambda_{i}$ is the deviation of the delay of the received signal from the calculated one, $\mathrm{n}$ is the additive noise, $\psi_{i}=\omega \lambda_{1}$.

For the recorded digitized video signal of the received pulse and the a priori known complex modulating function of the sounding signal, their correlation function (CF) is calculated 


$$
\dot{K}_{i m}=\sum_{k} \dot{\xi}_{i k} \cdot \dot{a}_{i} *\left((k-m) \cdot \tau_{s}\right),
$$

being a discrete expression of the continuous correlation function

$$
\dot{K}_{i}(\lambda)=\int_{-\infty}^{\infty} \dot{\xi}_{i}(\tau) \cdot \dot{a}_{i} *\left(\tau-\tau_{0 i}-\lambda\right) d \tau
$$

A coarse (up to an integer number of samples) determination of the maximum position of the correlation function sampled at the same frequency as the input signal is performed. A fragment of the correlation function within the limits of $\pm(8 \ldots 16)$ samples from the maximum is interpolated with coefficient of $16 \ldots 32$ to specify the determination of the relative delay of the received pulse.

The initial phase of the received pulse is defined as the phase of the complex CF value at the point corresponding to the specified delay value. When determining the specified phase value, $\mathrm{CF}$ interpolation is applied.

The discriminatory characteristic (DC) is constructed in the form of

$$
D(\lambda)=\frac{d}{d \lambda} \hat{K}(\lambda)
$$

where $\hat{K}(\lambda)$ is the estimated appearance of the main lobe of the sounding signal ACF or the function that is its approximation. The most common types of modulation are the chirp and the phase code modulation, the calculated ACF of which have the form of function $\sin x / x$ and a triangle, respectively. Given the difference between the ACF shape of the real signal and the calculated one, due to the limited bandwidth of the radar transmitter and receiver, it is advisable to use Gaussian $\hat{K}(\lambda)=\exp \left\{-\lambda^{2} / 2 \delta^{2}\right\}$ as a universal approximation, where $\delta$ is the calculated ACF width as a function of delay. In this case, DC takes the form of an odd function $D(\lambda)=-\lambda / \delta^{2} \cdot \exp \left\{-\lambda^{2} / 2 \delta^{2}\right\}$.

The block diagram of the active simulator [7] is shown in Fig. 3. To stabilize the delay and the initial phase of the simulated reflected pulse relative to the received sounding one, the following elements are provided: 1) a hardware - software analyzer of the sounding pulse, allowing to determine the displacement of its envelope and the deviation of the initial phase from the calculated one in real time (before the next pulse playback) and generate control codes to make appropriate corrections; 2 ) actuators - a controlled delay line and a phase shifter.

The accuracy of determining the delay and additional phase deviations should ensure, subject to subsequent compensation of these errors, the limitation of the noise introduced by the simulator into the generated return signal is not more than minus $40 \mathrm{~dB}$ relative to the level of the response function to a single point target in the resulting image. It is taken into account that errors in time and phase are determined, as a rule, by various hardware and software.

As for the principles of designing of an active simulator, it is considered that its purpose is flight testing and calibrating SARs of one's own design or those owned by the customer operator. The data provided by the SAR operator shall be used in the simulation of the reflected signal: the type of the sounding signal, the survey mode, the cyclogram of sensing, and the parameters of the carrier trajectory. All these data are taken into account when forming a reflected signal digital model array that is pre-recorded on the data storage. 


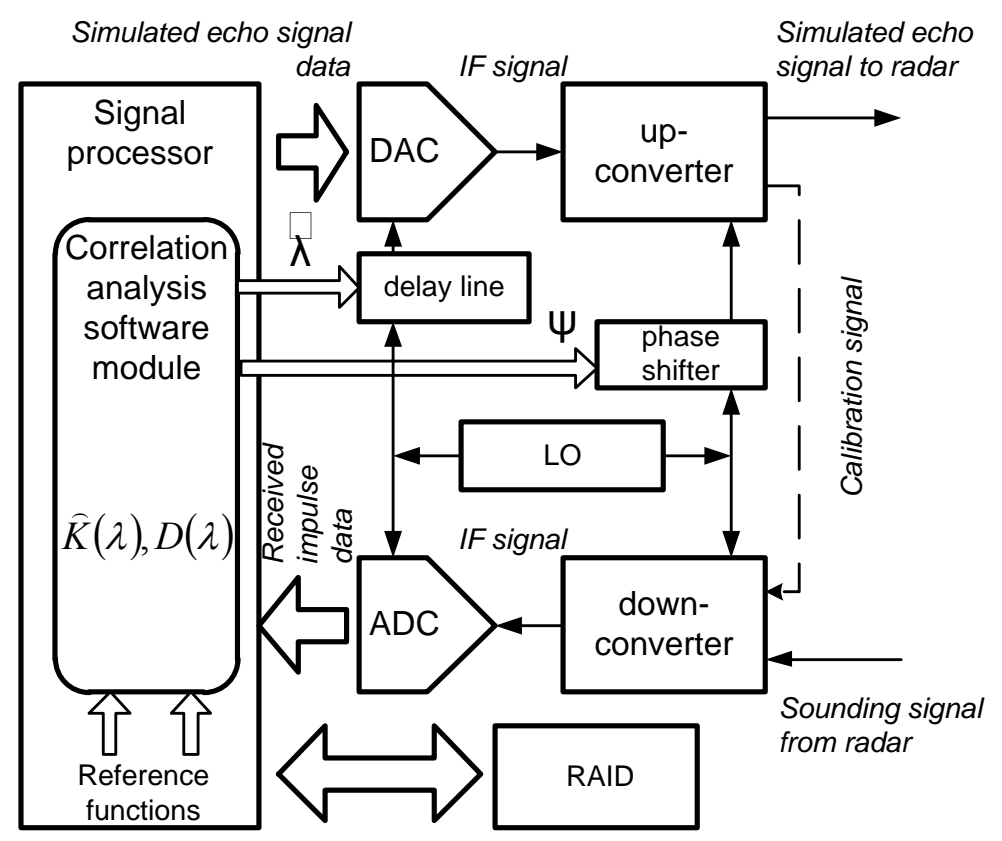

Fig. 3. The block diagram of the active simulator of arbitrary radar targets.

For the simulation of modulated sounding signal reflection from a point target considering the delay dependence on carrier movement, the combination of two hardware tools are used: rough (by values exceeding the range resolution) - step delay tuning by an integer number of clock cycles, fine - by retransmitted signal initial phase tuning.

The delay line and phase shifter are controlled by codes selected from the calibration table. For calibration a passive loop is provided in the simulator circuit at the carrier frequency between the transmitter output and the receiver input. Calibration consists in forming and transmitting a sample sounding pulse with the serial setting of all control codes of the delay line and zero phase shift, while the digitized signal from the output of the receiver is processed by a correlation analyzer to measure the actual delay and phase shift. The obtained values are stored in the correction code table. Similar calibration cycles are performed at multiple carrier frequencies in the operating range.

The active simulator is developed similarly to that used in the composition of the hardware and software complex of experimental tryout, described in [9]. Unlike its predecessor, the focus is on its operating in the absence of technological synchronization signals when working in an anechoic chamber with a simulated signal transmission directly to the antenna system, as well as on flight tests of space-based and airborne SARs.

Based on the mathematical description of the process of generating signals reflected from point and surface-distributed targets, a convolutional transformation algorithm is developed to be used in hardware-in-the-loop simulation to generate test impacts on the SAR in order to determine its spatial resolution and radiometric characteristics.

The proposed method for synchronizing an active simulator with an input sounding signal when generating a simulated reflected signal from a previously prepared digital model allows to simulate complex test patterns of large dimension and generate test impacts required for determining SAR radiometric characteristics.

The active simulator is used to determine spatial resolution and radiometric characteristics in hardware-in-the-loop simulation at the stage of ground tryout, and will also be used in flight tests and regular operation of space SARs using unified techniques, which will allow a systematic approach to the creation and operation of a radar system. 


\section{References}

1. V.S. Verba, A.R. Ilchuk, T.A. Lepekhina etc. Radar systems for aerospace monitoring of the Earth surface and airspace. Monograph / Ed. V.S. Verba, B.G. Tatarskiy (Moscow, Radioengineering. In Russian) (2014)

2. V.S. Verba, L.B. Neronsky, V.E. Turuk. Promising technologies for digital processing of radar information from space SAR (Moscow, Radioengineering, in Russian) (2019)

3. S. Raab, D. Rudolf, K. Weidenhaupt, M. Schwerdt. Development of DLR's Innovative Remote Controlled Calibration Targets - Potential of Polarization Sensitive Measurements. Proc. 12 European Conference on Synthetic Aperture Radar (EuSAR2018), pp. 69-74 (2018)

4. K. Weidenhaupt, S. Raab, D. Rudolf, M. Schwerdt. Innovative Antenna Concepts for DLR's Fully Polarimetric Low-Frequency Transponders. Proc. 12 European Conference on Synthetic Aperture Radar (EuSAR-2018), pp.83-86 (2018)

5. T. Lepekhina, V. Nikolaev, 2019 Russian Open Conference on Radio Wave Propagation (RWP), pp. 333-336 (2019)

6. T.A. Lepekhina, V.I. Nikolaev. Echo signal digital simulation algorithm for highresolution SAR tests. Processings of the 15-th International Conference "Digital signal processing and its applications" (Moscow, in Russian) (2013)

7. T.A. Lepekhina, V.I. Nikolaev. Synchronization of active radar target simulator for SAR tests Proc. VII All-Russia Science\&Technology Conference "Problems of Perspective Micro- and Nanoelectronic Systems Development" Proceedings, edited by A. Stempkovsky, Part 1. P. 236-241 (Moscow, IPPM RAS, 2016, in Russian) (2016)

8. I.N. Beloglazov, V.P. Tarasenko. Correlation-Extreme Systems (Moscow, Radio and Communications Publ, in Russian) (1974)

9. T.A. Lepekhina, V.I. Nikolaev, A.M. Tarasenko, "A hardware and software complex of experimental tryout for radioelectronic facility signal simulation, registration and analysis", Proc. VIII All-Russia Science\&Technology Conference "Problems of Perspective Micro- and Nanoelectronic Systems Development" (MES-2018), edited by A. Stempkovsky, part 4, pp. 119-126 (Moscow, IPPM RAS, in Russian) (2018) 